\title{
MODEL-BASED ADAPTIVE CONTROL OF ACETATE CONCENTRATION DURING THE PRODUCTION OF RECOMBINANT PROTEINS WITH E. COLI
}

\author{
I. Rocha, E.C. Ferreira \\ Centro de Engenharia Biológica - IBQF, Universidade do Minho \\ 4710-057 Braga, PORTUGAL \\ e-mail: \{irocha,ecferreira\}@deb.uminho.pt
}

\begin{abstract}
A model-based adaptive linearizing control law was derived for the regulation of the acetate concentration during the fed-batch fermentation of recombinant proteins with high cell density culture of Escherichia coli growing on glucose.

An unstructured model for the growth was applied to the major metabolic pathways: oxidative growth on glucose, fermentative growth on glucose, oxidative growth on acetate, and maintenance. A model order reduction method was used to allow the development of the control algorithm without the knowledge of the kinetic structure being necessary. The non-linear model was subjected to transformations in order to obtain a linear behaviour for the control loop when a non-linear control is applied.

The control law requires on-line acetate and carbon dioxide and oxygen transfer rates measurements. Acetate measurements are achieved with a developed Flow Injection Analysis (FIA) physical-chemical method. The gas transfer rates are calculated from gas analysis data obtained with a Mass Spectrometer (MS) connected to the exhaust gas line of the fermenter and also to the inlet aeration line. These calculations, as well as the implementation of the control law were performed through a MATLAB script embedded in a LABView program that also acquired data from the FIA system and other relevant state variables from the fermenter Digital Control Unit. Copyright (C) 2002 IFAC
\end{abstract}

Key-words: fermentation processes, adaptive control, model-based control, sensors, nonlinear systems, Lyapunov methods.

\section{INTRODUCTION}

High cell density fermentation of Escherichia coli with glucose as sole carbon source is the most widely used method for the production of recombinant proteins, due to the high knowledge of this microorganism and the high production yields obtainable (Lee, 1996).

However, this productivity can be affected by the presence of acetate, the major by-product of the glucose metabolism. Acetate is produced both when E. coli is grown under anaerobic or oxygen-limiting conditions and when carbon flux into the central metabolic pathways exceeds the biosynthetic demands and the capacity for energy generation within the cell (Han et al., 1992; Walle and Shiloach, 1998)

Among the consequences of that production are the decrease of the biomass yield, the inhibition of the growth when acetate is present at high concentrations and the decrease of the production of recombinant proteins (Riesenberg et al., 1991, Rothen et al., 1998).

For these reasons, the accurate control of the acetate concentration is a very important issue when trying to achieve high productivities with this kind of processes. 
The lack of a reliable sensor for the on-line monitoring of acetate concentrations explains why few works have been published in this field (Konstantinov et al., 1990; Akesson, et al., 1999)

Also, the non-linearity exhibited by these processes, coupled with the difficulties in model identification and the time varying properties of the model parameters is often a drawback in the application of model-based control strategies. To deal with these uncertainties, an adaptive approach of the modelbased controller can be an attractive alternative.

The main purpose of this work was to develop an adaptive algorithm for the on-line control of acetate during the fed-batch fermentation of recombinant E. coli, taking advantage of an experimental developed technique for the on-line measurement of this metabolite (Rocha and Ferreira, 2001).

This paper is organized as follows: in part 2, the model based adaptive control of the process is presented, including the description of the process model, the explanation of the method for model order reduction, and the derivation of the control and adaptation laws. In part 3, the main results obtained are presented. Part 4 contains the major conclusions extracted from this work.

\section{MODEL-BASED ADAPTIVE CONTROL}

\subsection{Process Modelling}

The general state space dynamical model described by Bastin and Dochain (1990) is accepted as representing the dynamics of an $n$ components and $m$ reactions bioprocess:

$\frac{d \xi}{d t}=K \varphi(\xi, t)-D \xi+F-Q$

where $\xi \in \mathbb{R}^{\mathrm{n}}$ is a vector representing the state components; $\mathbf{K} \in \mathbb{R}^{\mathrm{n} \times \mathrm{m}}$ is the yield coefficient matrix; $\varphi \in \mathbb{R}^{\mathrm{m}}$ is the growth rates vector; the vectors $\mathbf{F}$ and $\mathbf{Q}$ are the feed rates and the gaseous outflow rates, respectively $\left(\mathbf{F}, \mathbf{Q} \in \mathbb{R}^{\mathrm{n}}\right)$. The scalar $D$ is the dilution rate ( $D^{-1}$ is the residence time), which will be the manipulated variable, defined as follows:

$$
D(t)=\frac{F_{i n}(t)}{W(t)}
$$

being $F_{\text {in }}$ the influent flow rate $\left(\mathrm{kg} \mathrm{h}^{-1}\right)$, and $W$ the culture medium weight $(\mathrm{kg})$.

The growth of E. coli may be interpreted through four main reactions (Galvanauskas et al., 1998; Cockshott and Bogle, 1999):

Respiratory growth on glucose:

$$
k_{1} S+k_{5} O \stackrel{\varphi_{1}}{\rightarrow} X+k_{7} C
$$

Fermentative growth on glucose:

$$
k_{2} S \stackrel{\varphi_{2}}{\rightarrow} X+k_{8} C+k_{3} A
$$

Respiratory growth on acetate:

$$
k_{4} A+k_{6} O \stackrel{\varphi_{3}}{\rightarrow} X+k_{9} C
$$

Maintenance:

$S+k_{m 1} A+k_{m 2} O \stackrel{\varphi_{4}}{\rightarrow} k_{m 3} C$

where $S, O, X, C, A$ represent sugar (glucose), oxygen, biomass, carbon dioxide, and acetate respectively (in the sequel $S, O, X, C$, and $A$ mean concentrations); $\varphi_{1}, \varphi_{2}, \varphi_{3}$, and $\varphi_{4}$ are the growth rates; $k_{i}$ are the yield (stoichiometric) coefficients.

The application of the general state space dynamical model to these reaction network leads to the following model:

$\frac{d}{d t}\left[\begin{array}{c}X \\ S \\ A \\ O \\ C\end{array}\right]=\left[\begin{array}{cccc}1 & 1 & 1 & 0 \\ -k_{1} & -k_{2} & 0 & -1 \\ 0 & k_{3} & -k_{4} & -k_{m 1} \\ -k_{5} & 0 & -k_{6} & -k_{m 2} \\ k_{7} & k_{8} & k_{9} & k_{m 3}\end{array}\right]\left[\begin{array}{c}\varphi_{1} \\ \varphi_{2} \\ \varphi_{3} \\ \varphi_{4}\end{array}\right]-D\left[\begin{array}{c}X \\ S \\ A \\ O \\ C\end{array}\right]+\left[\begin{array}{c}0 \\ D S_{i n} \\ 0 \\ O T R \\ 0\end{array}\right]-\left[\begin{array}{c}0 \\ 0 \\ 0 \\ 0 \\ C T R\end{array}\right]$

where $C T R$ is the carbon dioxide transfer rate from liquid to gas phase, OTR is the oxygen transfer rate from gas to liquid phase, and $S_{i n}$ is the influent glucose concentration.

The E. coli fermentation process is accepted as occurring in two possible metabolic regimens (Galvanauskas et al., 1998), - i) a respirofermentative regime, corresponding to an acetate production state, and ii) a respirative regime, corresponding to an acetate consumption pathway. Model (7) can then be separated in two distinct models: 


$$
\begin{gathered}
\frac{d}{d t}\left[\begin{array}{c}
X \\
S \\
A \\
O \\
C
\end{array}\right]=\left[\begin{array}{ccc}
1 & 1 & 0 \\
-k_{1} & -k_{2} & -1 \\
0 & k_{3} & -k_{m 1} \\
-k_{5} & 0 & -k_{m 2} \\
k_{7} & k_{8} & k_{m 3}
\end{array}\right]\left[\begin{array}{l}
\varphi_{1} \\
\varphi_{2} \\
\varphi_{4}
\end{array}\right]-D\left[\begin{array}{c}
X \\
S \\
A \\
O \\
C
\end{array}\right]+\left[\begin{array}{c}
0 \\
D S_{i n} \\
0 \\
O T R \\
0
\end{array}\right]-\left[\begin{array}{c}
0 \\
0 \\
0 \\
0 \\
C T R
\end{array}\right] \\
\frac{d}{d t}\left[\begin{array}{c}
X \\
S \\
A \\
O \\
C
\end{array}\right]=\left[\begin{array}{ccc}
1 & 1 & 0 \\
-k_{1} & 0 & -1 \\
0 & -k_{4} & -k_{m 1} \\
-k_{5} & -k_{6} & -k_{m 2} \\
k_{7} & k_{9} & k_{m 3}
\end{array}\right]\left[\begin{array}{l}
\varphi_{1} \\
\varphi_{3} \\
\varphi_{4}
\end{array}\right]-D\left[\begin{array}{c}
X \\
S \\
A \\
O \\
C
\end{array}\right]+\left[\begin{array}{c}
0 \\
D S_{i n} \\
0 \\
O T R \\
0
\end{array}\right]-\left[\begin{array}{c}
0 \\
0 \\
0 \\
0 \\
C T R
\end{array}\right]
\end{gathered}
$$

\subsection{Model Order Reduction}

Given the difficulties associated with the determination of the kinetic structure, it is considered unknown throughout this paper. Thus, the parameter estimation of the adaptive controller will have to be based on a reformulated model free of the kinetic terms. In those cases where a number of fast dynamic state components are recognised, this number being equal to or larger than the number of unknown kinetic terms, it is possible to assume that such components are in pseudo steadystate and, through the single perturbation method, to reformulate the problem in terms of a reduced model.

Assuming that glucose, oxygen, and carbon dioxide exhibit fast dynamics relatively to the slow limiting dynamics of biomass and acetate, and adopting the single perturbation procedure, it is possible to obtain a reduced model representation of the process:

i) recurring to the algebraic equations for glucose, oxygen and carbon dioxide, growth rates $\left(\varphi_{1}, \varphi_{2}\right.$ and $\varphi_{4}$ in model (8) and $\varphi_{1}, \varphi_{3}$ and $\varphi_{4}$ in model (9)) are obtained as functions of vectors $\mathbf{F}$ and $\mathbf{Q}$;

ii) substituting these algebraic equations in the dynamic equations for biomass and acetate leads to a reduced representation of such dynamics. The dynamics of acetate can then be written as follows:

$$
\frac{d A}{d t}=-\theta_{1} C T R-\theta_{2} O T R+\theta_{3} D S_{i n}-D A
$$

where the parameters $\theta_{1}, \theta_{2}$, and $\theta_{3}$ are variable functions of the yield coefficients, depending at each moment on the prevalent metabolic regime. The theoretical dependency of these variables on the yield coefficients for each case is represented in table 1. A priori knowledge from those coefficients was used as initial values in the experiments.
Table 1 Theoretical dependency of the parameters $\theta$

\begin{tabular}{lll}
\hline & \multicolumn{1}{c}{$\begin{array}{c}\text { Respiro- } \\
\text { fermentative }\end{array}$} & \multicolumn{1}{c}{ Respirative } \\
\hline$\theta_{1} \times \mathrm{K}^{\prime}$ & $\mathrm{k}_{1} \mathrm{k}_{3} \mathrm{k}_{\mathrm{m} 2}-\mathrm{k}_{3} \mathrm{k}_{5}$ & $\mathrm{k}_{4} \mathrm{k}_{5}-\mathrm{k}_{1} \mathrm{k}_{6} \mathrm{k}_{\mathrm{m} 1}-\mathrm{k}_{1} \mathrm{k}_{4} \mathrm{k}_{\mathrm{m} 2}$ \\
$\theta_{2} \times \mathrm{K}^{\prime}$ & $\mathrm{k}_{3} \mathrm{k}_{7}-\mathrm{k}_{1} \mathrm{k}_{3} \mathrm{k}_{\mathrm{m} 3}$ & $\mathrm{k}_{1} \mathrm{k}_{4} \mathrm{k}_{\mathrm{m} 3}-\mathrm{k}_{4} \mathrm{k}_{7}-\mathrm{k}_{1} \mathrm{k}_{9} \mathrm{k}_{\mathrm{m} 1}$ \\
$\theta_{3} \times \mathrm{K}^{\prime}$ & $\mathrm{k}_{3} \mathrm{k}_{7} \mathrm{k}_{\mathrm{m} 2}-\mathrm{k}_{3} \mathrm{k}_{5} \mathrm{k}_{\mathrm{m} 2}$ & $\mathrm{k}_{4} \mathrm{k}_{5} \mathrm{k}_{\mathrm{m} 3}-\mathrm{k}_{4} \mathrm{k}_{7} \mathrm{k}_{\mathrm{m} 2^{-}}$ \\
& & $\mathrm{k}_{5} \mathrm{k}_{9} \mathrm{k}_{\mathrm{m} 1}+\mathrm{k}_{6} \mathrm{k}_{7} \mathrm{k}_{\mathrm{m} 1}$ \\
$\mathrm{~K}^{\prime}$ & $\mathrm{k}_{5} \mathrm{k}_{8}+\mathrm{k}_{2} \mathrm{k}_{7} \mathrm{k}_{\mathrm{m} 2^{-}}$ & $\mathrm{k}_{1} \mathrm{k}_{6} \mathrm{k}_{\mathrm{m} 3}+\mathrm{k}_{1} \mathrm{k}_{9} \mathrm{k}_{\mathrm{m} 2}+\mathrm{k}_{5} \mathrm{k}_{9^{-}}$ \\
& $\mathrm{k}_{1} \mathrm{k}_{8} \mathrm{k}_{\mathrm{m} 2}-\mathrm{k}_{2} \mathrm{k}_{5} \mathrm{k}_{\mathrm{m} 3}$ & $\mathrm{k}_{6} \mathrm{k}_{7}$ \\
\hline
\end{tabular}

\subsection{Adaptive Control}

The objective of the control algorithm will be to control the variable $A$, which will follow a reference value represented by $A^{*}(t)$. The linearizing control problem consists of deducing and implementing a non-linear law $D$ as a function of $\xi, \mathbf{Q}$, and $A^{*}$, such that the controller convergence error $\tilde{A}=\left(A^{*}-A\right)$ can be governed by a pre-specified stable linear differential equation, known as reference model. The control objective will consist of imposing a stable first order closedloop dynamics of the form:

$$
\frac{d}{d t}\left(A^{*}-A\right)+\lambda\left(A^{*}-A\right)=0, \lambda>0
$$

where $\lambda$ is the controller gain. Combining the inputoutput reduced model (10) with the reference model (11), will easily lead to the following linearizing regulation law:

$$
D(t)=\frac{\theta_{1} C T R+\theta_{2} O T R+\lambda_{1}\left(A^{*}-A\right)}{\theta_{3} S_{i n}-A}
$$

The discretized form of the regulation law can be written as follows:

$$
D_{k}=\frac{\theta_{1, k} C T R_{k}+\theta_{2, k} O T R_{k}+\lambda_{1}\left(A^{*}-A_{k}\right)}{\theta_{3, k} S_{i n}-A_{k}}
$$

Employing the reduced model, will lead to concentrate all the unknowns in the $\theta$ parameters, which will be estimated on-line. Hence, in eqs. (12) and (13), the estimates $\hat{\theta}$ will be employed rather than the true values $\theta$.

\subsection{Estimation Laws}

The basis for the method adopted is the so-called Lyapunov design as described by Bastin and Dochain (1990). It is assumed that each parameter $\theta_{i}$ is estimated by the following adaptive law: 
$\frac{d \hat{\theta}_{i}}{d t}=-\gamma \phi_{i}^{-1}\left(A^{*}-A\right)$

where $\phi_{1}$ is the regressor associated to $\theta_{i}$, depending on $\mathbf{F}$ and $\mathbf{Q}$, and $\gamma$ a positive definite estimator gain. This equation is consequence of fixing the closed loop dynamics by choosing a real double pole, as proposed by Perrier and Dochain (1993), keeping coupled controller and estimator parameter adaptation. Based on this approach Ferreira and Feyo de Azevedo (1996) proposed a tuning scheme that enforces a second order convergence dynamics with gains computed as:

$\lambda \equiv \frac{2 \zeta}{\tau}-\frac{1}{\phi_{i}} \frac{d \phi_{i}}{d t}$

$\gamma \equiv \frac{1}{\tau^{2}}$

which has the meaning that each $\hat{\theta}_{i}$ converges for the true $\theta_{i}$ through a $2^{\text {nd }}$ order dynamic trajectory, with a natural period of oscillation $\tau$ and a damping coefficient $\zeta$.

In the discretized form, this adaptation law can be written as follows:

$\hat{\theta}_{1, k+1}=\hat{\theta}_{1, k}+T \frac{A^{*}-A_{k}}{C T R_{k} \tau^{2}}$

$\hat{\theta}_{2, k+1}=\hat{\theta}_{2, k}+T \frac{A^{*}-A_{k}}{O T R_{k} \tau^{2}}$

$\hat{\theta}_{3, k+1}=\hat{\theta}_{3, k}+T \frac{A^{*}-A_{k}}{D_{k} S_{i n} \tau^{2}}$

being $T$ the sampling period and $k$ a time index.

\section{EXPERIMENTAL SETUP}

\subsection{Fermentation Conditions}

The experimental conditions for the fermentation process are described elsewhere (Rocha and Ferreira, 2001). The Flow Injection Analysis (FIA) system (Ismatec, Switzerland) employed for the online measurement of acetate is shown in figure 1. It was adapted from Forman et al. (1991) and is based on the diffusion of acetate through a gas-diffusion chamber into a stream containing an acid-base indicator. The subsequent decrease in the absorbance is detected with an incorporated photometer.
The gas transfer rates are calculated from gas analysis data obtained with a Mass Spectrometer connected to the exhaust gas line of the fermenter and also to the inlet aeration line.

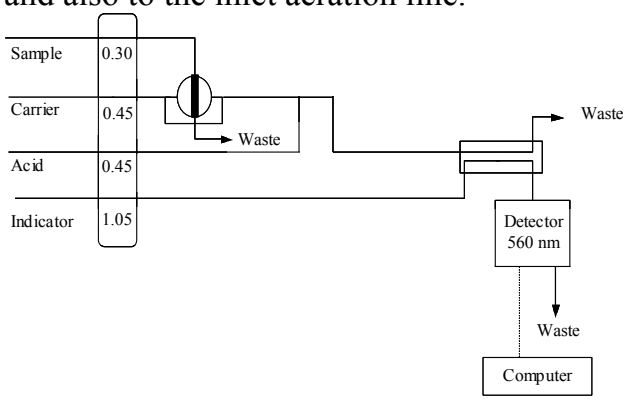

Fig. 1. Schematic representation of the system used for the analysis of acetate with FIA.

\subsection{Hardware and Software}

OTR and CTR calculations, as well as the implementation of the control law were performed through a MATLAB (version 6.0) script imbedded in a LABView (version 6.0) program that also acquired data from the FIA system and other relevant state variables from the fermenter Digital Control Unit. The simulations for the optimization of the parameters used in the control algorithm were run with Compaq Visual Fortran 6.1.

\section{RESULTS}

The results presented in this section are based on simulations of the model described in section 2.1 with values obtained from Galvanauskas et al. (1998). The control algorithm derived in section 2.3 and 2.4 was used to control acetate concentration. The chosen set-point was $0.5 \mathrm{~g} / \mathrm{kg}$ (below inhibition levels).

The sampling period for the simulations has to be compatible with both Mass Spectrometer and Flow Injection Analysis measurements. In the first case, the sampling rate is relatively high ( 3 samples per minute), while the FIA system allows acetate measurement every 2 minutes. The results shown were obtained with a sampling period of $0.05 \mathrm{~h}(3$ minutes).

An example of the fermentation behaviour is shown in figure 2. Due to the control of acetate concentration, the microorganism is able to grow without acetate inhibition until the end of the fedbatch. The final biomass concentration achieved was $22 \mathrm{~g} / \mathrm{kg}$, representing approximately $33 \mathrm{~g} / \mathrm{L}$. Glucose accumulation is also very limited. Control action is also shown in figure 2 . 
For the adaptive control algorithm, different approaches were studied, namely the simultaneous estimation of the three $\theta$ parameters; the fixation of one $\left(\theta_{3}\right)$, and two $\left(\theta_{2}\right.$ and $\left.\theta_{3}\right)$ of them, based on the theoretical dependency on the yield coefficients described in table 1. Comparative results are shown in figure 3. In all cases, a good convergence of the controlled variable to the set-point is obtained. However, a slightly smaller divergence occurs at the beginning of the fermentation when the three parameters are adapted on-line. A good convergence of the estimated parameters was obtained in all experiments (results not shown).
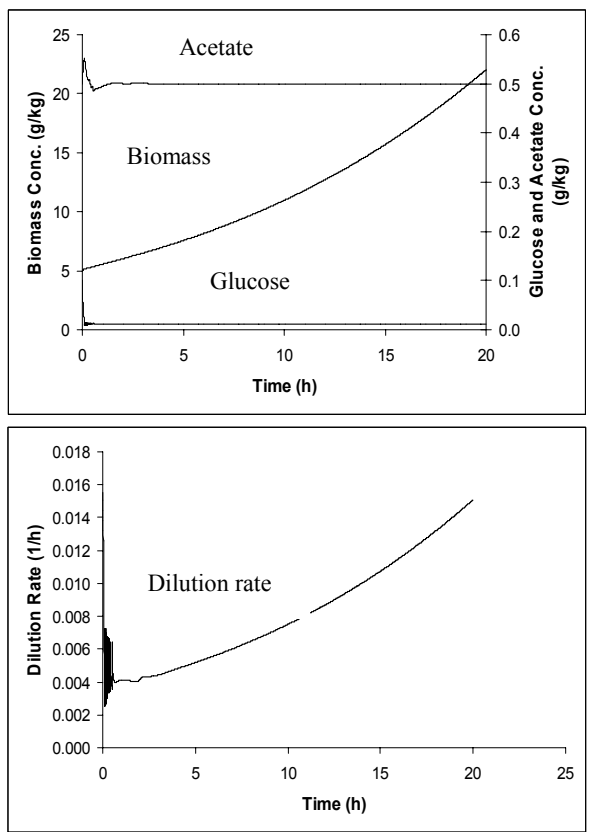

Fig. 2. Example of a controlled fermentation.

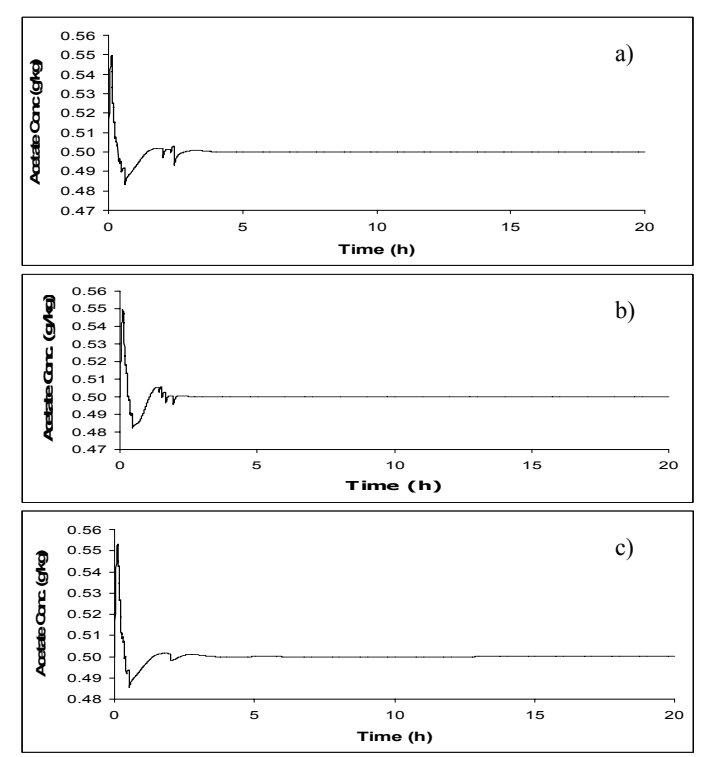

Fig. 3. Fixing $\theta_{2}$ and $\theta_{3}$ (a), fixing only $\theta_{3}(\mathrm{~b})$, and estimating the three $\theta(\mathrm{c})$.
In order to test the controller's robustness, other simulations were performed. The acetate set-point was switched from 0.5 to $1 \mathrm{~g} / \mathrm{kg}$ at $10 \mathrm{~h}$ of fermentation run, with a good set-point tracking behaviour (figure 4) while keeping the same tuning parameters.

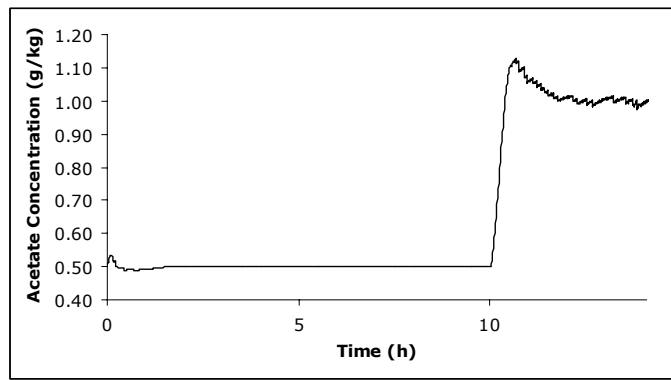

Fig. 4. Set-point tracking from 0.5 to $1 \mathrm{~g} / \mathrm{kg}$.

A $50 \%$ change in the glucose concentration in the feeding at $10 \mathrm{~h}$ was also studied. Figure 5 shows the results obtained in this test. A small decrease in acetate concentration is verified, although the controller rapidly imposes a convergence to the setpoint.

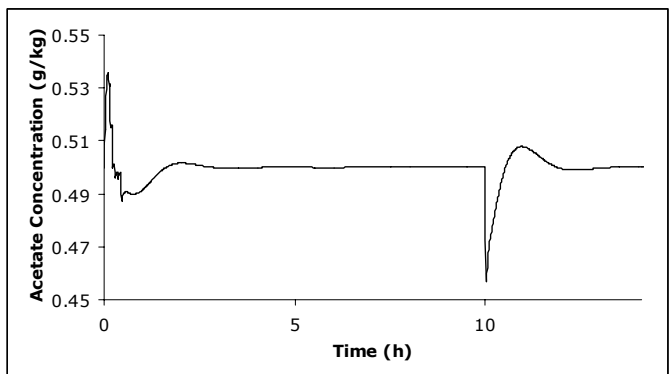

Fig. 5. Effects of a $50 \%$ change in the $S_{\text {in }}$ at $10 \mathrm{~h}$.

In order to check for the effect of signal to noise ratio on the controller performance, a 5\% white noise was added to the measured variables (acetate and exhausted oxygen and carbon dioxide). It is clear from figure 6 that the controller still converges to the set-point, although the variance increases as the fermentation approaches the end.

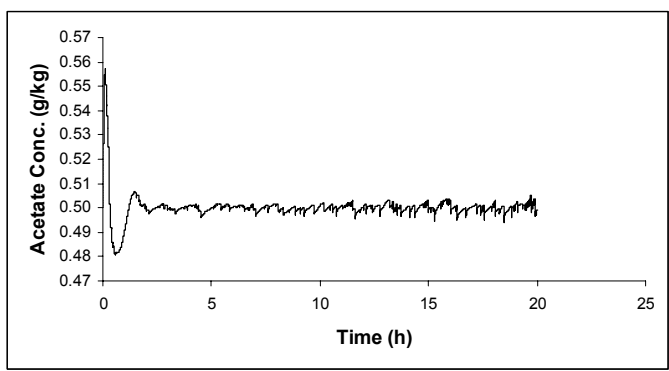

Fig. 6. Effect of a 5\% signal to noise in measured variables. 


\section{CONCLUSIONS}

An algorithm for the on-line adaptive control of the undesirable acetate production during the recombinant $E$. coli fed-batch fermentation was developed. It is based in the application of Lyapunov estimation design for parameter adaptation of a linearizing regulation algorithm using a reduced version of the state space dynamical model.

Simulation studies indicate that the developed algorithm is able to maintain acetate below toxic levels, converging rapidly to the imposed set-point.

The controller's robustness was assessed by changing the set-point and the influent glucose concentration, as well as introducing a 5\% noise signal in the measured variables. The responses obtained were considered satisfactory.

The simulation results obtained give encouraging indications towards the real process implementation of the algorithm. The existence of methods for the on-line analysis of acetate (a developed FIA system) and for the on-line determination of CTR and $O T R$ makes this real-time implementation straightforward.

\section{ACKNOWLEDGEMENTS}

This work was supported by the PROTEXPRESS project (Agência de Inovação). Fundação para a Ciência e a Tecnologia provided financial support for I. Rocha through a doctoral research grant (PRAXIS XXI/BD/16961/98).

\section{REFERENCES}

Akesson, M.; E. N. Karlsson, P. Hagander, J. P. Axelsson, and A. Tocaj (1999). On-line detection of acetate formation in Escherichia coli cultures using dissolved oxygen responses to feed transients. Biotechnology and Bioengineering, 64, 590-598.

Bastin, G., and D. Dochain (1990). On-line Estimation and Adaptive Control of Bioreactors. Elsevier Science Publishers, Amsterdam.

Cockshott, A. and I. Bogle (1999). Modelling the effect of glucose feeding on a recombinant $E$. coli fermentation. Bioprocess Engineering, 20, 83-90.

Ferreira, E.C., S. Feyo de Azevedo (1996). Adaptive Linearizing Control of Bioreactors. In: UKACC Int. Conf CONTROL'96,
Conference. Conference Publication No. 427, Vol. II, 1184-1189. (IEE), Exeter

Forman, L. W., B. D. Thomas and F. S. Jacobson (1991). On-line monitoring and control of fermentation processes by flow-injection analysis. Analytica Chimica Acta, 249, 101111.

Galvanauskas, V., R. Simutis, N. Volk and A. Lübert (1998). Model Based Design of a Biochemical cultivation process. Bioprocess Engineering, 18, 227-234.

Han, K., H. Lim and J. Hong (1992). Acetic Acid Formation in Escherichia coli Fermentation. Biotechnology and Bioengineering, 39, 663671.

Konstantinov, K., M. Kishimoto, T. Seki, and T. Yoshida (1990). A balanced DO-Stat and its application to the control of acetic acid excretion by recombinant Escherichia coli. Biotechnology and Bioengineering, 36, 750758

Lee, S. Y. (1996). High cell-density culture of Escherichia coli. Trends in Biotechnology, 14, 98-105.

Perrier, M., and D. Dochain (1993). Evaluation of Control Strategies for Anaerobic Digestion Processes. Int. J. Adaptive Control and Signal Processing, 7, 309-321.

Riesenberg, D., V. Schulz, W.A. Knorre, H.-D. Pohl, D. Korz, E. A. Sanders, A. Rob and W.D. Deckwer (1991). High Cell Density Cultivation of Escherichia coli at Controlled Specific Growth Rate. Journal of Biotechnology, 20, 17-28.

Rocha, I. and E. C. Ferreira (2001). An Integrated System for Advanced Monitoring and Control of Fed-batch Fermentations of Recombinant $E$. coli In:Proceedings of the 8th International Conference in Computer Applications in Biotechnology. (D. Dochain, M. Perrier eds). 353-358. Quebec, Canada.

Rothen, S. A., M. Sauer, B. Sonnleitner and B. Witholt (1998). Growth Characteristics of Escherichia coli HB101[pGEc47] on Defined Medium. Biotechnology and Bioengineering, 58, 92-100.

Walle, M. and J. Shiloach (1998). Proposed Mechanism of Acetate Accumulation in Two Recombinant Escherichia coli Strains during High Density Fermentation. Biotechnology and Bioengineering, 57, 71-78. 